PROCEEDINGS OF THE

AMERICAN MATHEMATICAL SOCIETY

Volume 133, Number 9, Pages 2637-2646

S 0002-9939(05)07865-2

Article electronically published on March 15, 2005

\title{
FRAGMENTABILITY OF SEQUENCES OF SET-VALUED MAPPINGS WITH APPLICATIONS TO VARIATIONAL PRINCIPLES
}

\author{
MARC LASSONDE AND JULIAN P. REVALSKI
}

(Communicated by Jonathan M. Borwein)

\begin{abstract}
We propose to study fragmentability of set-valued mappings not only for a given single mapping, but also for a sequence of mappings associated with the initial one. It turns out that this property underlies several variational principles, such as for example the Deville-Godefroy-Zizler variational principle and the Stegall variational principle, by providing a common path for proof.
\end{abstract}

\section{INTRODUCTION AND PRELIMINARIES}

Let $T: Z \rightrightarrows X$ be a set-valued mapping between a topological space $Z$ and a metric space $(X, d)$. The mapping $T$ is said to be fragmented by $d$ (see [HJT]) if for any nonempty open set $U$ of $Z$ and every $\varepsilon>0$ there is a nonempty open set $U^{\prime} \subset U$ such that $d-\operatorname{diam} T\left(U^{\prime}\right)<\varepsilon$. This notion, which has turned out to be very useful in the study of the points of single-valuedness of set-valued mappings, is closely related to (and also motivated by) two other notions: fragmentability of topological spaces and minimal set-valued mappings.

Recall that a topological space $X$ is said to be fragmented by a metric $d$ in $X$ (see [JRo]) if for any nonempty set $A$ of $X$ and every $\varepsilon>0$, there is a nonempty open (in the original topology) set $V$ of $X$ such that $d-\operatorname{diam}(V \cap A)<\varepsilon$. Evidently, every metric space is fragmented by its own metric. But there are interesting cases of nonmetrizable topological spaces that are fragmentable by some metric. A typical situation is the case of certain real Banach spaces $X$ with its weak topology (or dual Banach spaces with the weak star topology) which can be fragmented by the norm: for the study of fragmentability see e.g. [JNRo, Ri1, Ri2, KMo].

Further, a set-valued mapping $T: Z \rightrightarrows X$ between topological spaces $Z$ and $X$ is said to be minimal if for any open sets $U$ of $Z$ and $V$ of $X$ so that $T(U) \cap$ $V \neq \emptyset$, there exists a nonempty open set $U^{\prime} \subset U$ with the property $T\left(U^{\prime}\right) \subset V$. This definition is motivated by the fact that the latter property characterizes the minimal (in the sense of graph inclusion) mappings $T: Z \rightrightarrows X$ which are usco in $Z$ (upper semicontinuous with nonempty compact values) ([ChrK $]$ ). However, there are important cases of mappings which are not necessarily usco but still possess the

Received by the editors April 20, 2004.

2000 Mathematics Subject Classification. Primary 49J53; Secondary 46B20, 46B22, 54C60.

The second author's research was supported by a Marie Curie Fellowship of the European Community program IHP under contract HPMF-CT-2002-01874.

(C)2005 American Mathematical Society Reverts to public domain 28 years from publication 2637 
above minimality property, such as for example the solution mapping related to minimization of continuous bounded functions in a topological space $X$, or metric projections in certain Banach spaces (see [ČKR2]).

It is straightforward to check that if $T: Z \rightrightarrows X$ is minimal and $X$ is fragmented by a metric $d$, then $T$ is fragmented by $d$ as well. Moreover, it is well known that a fragmentable mapping $T$ between a Baire space $Z$ and a space $X$ (with fragmenting metric $d$ ) is generically no more than single-valued. The latter means that there is a dense $G_{\delta}$-subset $Z_{1}$ of $Z$ such that for any $z \in Z_{1}$ either $T(z)=\emptyset$ or $T$ is single-valued at $z$. These facts have been used in proving, for instance, generic single-valuedness of maximal monotone mappings, in particular, generic differentiability of convex functions (cf. $[\mathrm{Ph}]$ ).

The above approach is suitable for mappings that are nonempty-valued. But, often one is faced with a situation where a set-valued mapping to be used may have empty values. This is a typical situation in several well known variational principles, where the general scheme is the following one: one is given a lower semicontinuous extended real-valued and bounded from below function $f: X \rightarrow \mathbb{R} \cup\{+\infty\}$ which is defined on a topological space $X$ and which is proper. The latter means that the effective domain $\operatorname{dom} f$ of $f$, i.e., the set $\operatorname{dom} f:=\{x \in X: f(x)<+\infty\}$ is nonempty. One possesses also a family $Z$ of usually continuous and bounded from below on $X$ functions $g: X \rightarrow \mathbb{R}$. The question is to investigate what is the set $Z_{1}:=\{g \in Z: f+g$ attains its minimum in $X$ (or stronger: the perturbed minimization problem $(X, f+g)$ is well-posed $)\}$.

Recall that a minimization problem $(X, h)$, with $h$ proper in $X$, is well-posed if there is a unique minimum of $h$ in $X$ towards which converges any minimizing sequence for $(X, h)$, i.e., any sequence $\left\{x_{n}\right\}_{n \geq 1} \subset X$ with $h\left(x_{n}\right) \rightarrow \inf h:=\inf _{X} h$. If $(X, h)$ is well-posed, then every minimizing net for $(X, h)$ also converges to the unique solution. The well-posedness of $(X, h)$ is also termed as that $h$ attains its strong minimum in $X$. The first aim in a typical variational principle is to prove that the set of good perturbations $Z_{1}$ is nonempty. If $Z$ is equipped with a complete metric, a further aim is to show that $Z_{1}$ is residual in $Z$, which means that it contains a dense $G_{\delta}$-subset of $Z$ (equivalently, $Z \backslash Z_{1}$ is of the first Baire category in $Z$ ).

The above scheme has been the case in the Ekeland variational principle [Ek1. Ek2, Borwein-Preiss smooth variational principle [BPr], Stegall variational principle $\overline{\mathrm{St}}$, the generic variational principle of Coban, Kenderov and Revalski [Č ČKR1, Deville-Godefroy-Zizler variational principle DGZ1 DGZ2 (see also a strengthening of the latter in $[\overline{D R}]$ ) and Ioffe-Zaslavski principle [IZa]. With the exception of the first two principles, in the remaining ones the set of good perturbations contains a dense $G_{\delta}$-subset of the space of all perturbations.

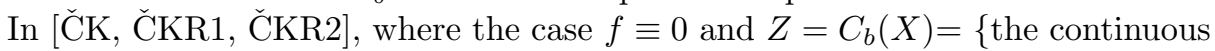
bounded functions in $X\}$, equipped with the uniform norm $\|g\|_{\infty}:=\sup \{|g(x)|$ : $x \in X\}$, was considered, an approach was proposed via the solution mapping $M: C_{b}(X) \rightarrow X$ assigning to each $g \in C_{b}(X)$ the set of minimum points to $g$. This mapping is minimal and due to the fact that it is densely nonempty, the corresponding generic principle was deduced via the existence of generically defined selections of $M$ ([СKR2]). Unfortunately, the latter approach cannot be used directly in the case of the Stegall principle, neither in the Deville-GodefroyZizler nor in the Ioffe-Zaslavski principle, because in these principles one does not 
know a priori (neither is it easily seen) whether the corresponding solution mapping is densely nonempty. In all these three principles the proofs have been based on a direct construction of a countable family of open dense subsets which gives as an intersection a dense $G_{\delta}$-subset of good perturbations.

We show in this paper, however, that behind these principles there is something common, of topological nature, which allows us to avoid this repeated construction in each case. Namely, we propose below, instead of studying a single mapping $T$ (corresponding to the solution mapping), to consider together with it a sequence of associated mappings $\left\{T_{n}\right\}_{n \geq 1}$ (which will correspond to the approximate solutions and) which give as an intersection the mapping $T$. We give a definition for the fragmentability of the sequence $\left\{T_{n}\right\}_{n \geq 1}$ and prove that in this case the initial mapping $T$ is single-valued and possesses a continuity-like property (with respect to the fragmenting metric) at the points of a dense $G_{\delta}$-subset of the domain space, provided the fragmenting metric is complete. The result is then applied to obtain by a common scheme the Deville-Godefroy-Zizler principle, the generic variational principle of Čoban, Kenderov and Revalski and the Ioffe-Zaslavski principle. Stegall's variational principle is also shown to fit this general scheme.

Let us mention, finally, that a strengthening of the Borwein-Preiss variational principle in Banach spaces which could be used to derive the Deville-GodefroyZizler principle is provided by Loewen and Wang in [LW. A one-perturbation variational principle (i.e. when $Z$ consists of a single function) with applications to differentiability in Banach spaces is studied in the recent paper [BCFR].

In the sequel, given a metric space $(X, d)$, as usual $B_{X}(x, r)$ means the open ball in $X$ around $x \in X$ with radius $r>0$, and for a set $A \subset X, d-\operatorname{diam} A$ is its diameter. All topological spaces in this article are supposed to be at least Hausdorff.

\section{Fragmentability of Sequences of mappings}

Let $\left\{T_{n}: Z \rightrightarrows X\right\}_{n \geq 1}$ be a decreasing sequence of nonempty-valued mappings between a topological space $Z$ and a metric space $(X, d)$. In other words, the sequence $\left\{T_{n}\right\}_{n \geq 1}$ satisfies $\emptyset \neq T_{n+1}(z) \subset T_{n}(z)$ for every $z \in Z$ and $n \geq 1$. Given such a sequence, we will denote by $T(z):=\bigcap_{n=1}^{\infty} T_{n}(z), z \in Z$, the limit (intersection) mapping generated by $\left\{T_{n}\right\}_{n \geq 1}$. Observe that the limit mapping $T$ may have empty values.

Definition 2.1. We say that the sequence $\left\{T_{n}: Z \rightrightarrows(X, d)\right\}_{n \geq 1}$ is fragmented by the metric $d$ if for every nonempty open set $U$ in $Z$ and $\varepsilon>0$ there are $n^{\prime} \geq 1$ and a nonempty open set $U^{\prime} \subset U$ so that $d-\operatorname{diam} T_{n^{\prime}}\left(U^{\prime}\right)<\varepsilon$.

In this case we also say that $d$ fragments the sequence $\left\{T_{n}\right\}_{n \geq 1}$. Evidently, if $d$ fragments the sequence, it fragments also the limit mapping $T$.

The proof of the next theorem follows the general path of proving generic singlevaluedness of a given (single) fragmentable mapping (see e.g. [ChrK, Ri1, $\mathrm{Ph}$ ). Recall that a Baire space is a topological space in which the intersection of a countable family of open and everywhere dense subsets is dense in the space.

Theorem 2.2. Let $\left\{T_{n}\right\}_{n \geq 1}$ be a decreasing sequence of nonempty-valued mappings between a Baire topological space $Z$ and a metric space $(X, d)$. Suppose that $d$ fragments $\left\{T_{n}\right\}_{n \geq 1}$. Then there is a dense $G_{\delta}$-subset $Z_{1}$ of $Z$ such that for any 
point $z \in Z_{1}$ the limit mapping $T$ satisfies:

(a) either $T(z)=\emptyset$, or

(b) $T(z)$ is a singleton, say $x$, and moreover, for any $\varepsilon>0$ there exist an open set $U \ni z$ and $n^{\prime} \geq 1$ with $T_{n^{\prime}}(U) \subset B_{X}(x, \varepsilon)$.

If the metric $d$ is complete and the values $T_{n}(z)$ are closed in $X$ for any $n$ and $z \in Z$, then each $z \in Z_{1}$ satisfies (b).

Proof. For each $k=1,2, \ldots$, consider the sets:

$$
W_{k}:=\bigcup\left\{U: U \neq \emptyset \text { open in } Z \quad \& \quad \exists n \geq 1: d-\operatorname{diam} T_{n}(U)<1 / k\right\} .
$$

Every $W_{k}$ is open. We show that each of these sets is also dense in $Z$. Let $k \geq 1$ be fixed and take an arbitrary nonempty open set $U$ in $Z$. By the fragmentability of $\left\{T_{n}\right\}_{n \geq 1}$ there are $n^{\prime} \geq 1$ and a nonempty open $U^{\prime} \subset U$ so that $d-\operatorname{diam} T_{n^{\prime}}\left(U^{\prime}\right)<$ $1 / k$. Evidently, $U^{\prime} \subset W_{k}$.

Let $Z_{1}:=\bigcap_{k=1}^{\infty} W_{k}$. This is a dense $G_{\delta}$-subset of $Z$. Fix some $z \in Z_{1}$. Then there exist a sequence of open sets $\left\{U_{k}\right\}_{k \geq 1}$ and a strictly increasing sequence of integers $\left\{n_{k}\right\}_{k \geq 1}$, so that for any $k \geq 1$ we have $z \in U_{k}$ and $d-\operatorname{diam} T_{n_{k}}\left(U_{k}\right)<1 / k$. Obviously, $T(z)=\bigcap_{k=1}^{\infty} T_{n_{k}}(z) \subset \bigcap_{k=1}^{\infty} T_{n_{k}}\left(U_{k}\right)$. The latter set is no more than a singleton. Suppose that $T(z) \neq \emptyset$. Then there is $x \in X$ so that

$$
\{x\}=T(z)=\bigcap_{k=1}^{\infty} T_{n_{k}}(z)=\bigcap_{k=1}^{\infty} T_{n_{k}}\left(U_{k}\right) .
$$

Observe that if the metric $d$ is complete and $T_{n}(z)$ are closed in $X$ for any $n \in \mathbb{N}$, then by Cantor's theorem we always have (1) fulfilled for the chosen $z$.

Let $\varepsilon>0$ be arbitrary. Take $k \geq 1$ so that $1 / k<\varepsilon$ and put $U:=U_{k}$ and $n^{\prime}:=n_{k}$. Since $\operatorname{diam} T_{n_{k}}\left(U_{k}\right)<1 / k$, we conclude by (1) that $T_{n^{\prime}}(U) \subset B_{X}(x, \varepsilon)$. The proof is completed.

Remark 2.3. Let us recall that a set-valued mapping $T: Z \rightrightarrows X$ between topological spaces $Z$ and $X$ is said to be upper semicontinuous at $z_{0} \in Z$ if for any open set $V \subset X$ such that $T\left(z_{0}\right) \subset V$ there is an open set $U \subset Z$ with $z_{0} \in U$ so that $T(U) \subset V$. Therefore, by (b) above, we see that $T$ is single-valued and upper semicontinuous with respect to the metric $d$ in $X$ at any $z \in Z_{1}$ for which $T(z)$ is nonempty. In particular, if the metric $d$ is complete and the values $T_{n}(z)$ are closed for any $n$ and $z$, then $T$ is single-valued and upper semicontinuous at any point of $Z_{1}$. These two properties are well known for a single fragmentable mapping (for the latter property the mapping must be with nonempty values everywhere). What is new in Theorem 2.2 is, first the continuity property in (b) which, in general, is stronger than the upper semicontinuity of the limit mapping and which is important for some of the applications below. We may express this property as that the sequence $\left\{T_{n}\right\}_{n \geq 1}$ is upper semicontinuous at $z$. The second contribution of the latter theorem is that we obtain the generic single-valuedness of the limit mapping without supposing that this mapping has nonempty values. This again (see the next section) is important for the applications where, as a rule, the corresponding solution mapping may have empty values.

Call the sequence $\left\{T_{n}: Z \rightrightarrows X\right\}_{n \geq 1}$ of mappings between the topological spaces $Z$ and $X$ minimal if for any nonempty open sets $U$ of $Z$ and $V$ of $X$ such that 
$T_{n}(U) \cap V \neq \emptyset$ for some $n \geq 1$, there are a nonempty open set $U^{\prime} \subset U$ and $n^{\prime} \in \mathbb{N}$ so that $T_{n^{\prime}}\left(U^{\prime}\right) \subset V$.

Evidently, if $\left\{T_{n}\right\}_{n \geq 1}$ is minimal and the space $X$ is fragmented by a metric $d$, then $\left\{T_{n}\right\}_{n \geq 1}$ is fragmented by $d$ as well. Therefore, the following is an immediate corollary of Theorem 2.2 .

Corollary 2.4. Let $\left\{T_{n}\right\}_{n \geq 1}$ be a decreasing sequence of nonempty-valued mappings between a Baire topological space $Z$ and a topological space $X$. Suppose that $X$ is fragmented by a metric $d$. Then, if $\left\{T_{n}\right\}_{n \geq 1}$ is minimal, there exists a dense $G_{\delta^{-}}$ subset $Z_{1}$ of $Z$ such that for any point $z \in Z_{1}$ the limit mapping $T$ satisfies:

(a) either $T(z)=\emptyset$, or

(b) $T(z)$ is a singleton, say $x$, and moreover, for any $\varepsilon>0$ there exist an open set $U \ni z$ and $n^{\prime} \geq 1$ with $T_{n^{\prime}}(U) \subset B_{X}(x, \varepsilon)$.

If the metric $d$ is complete, and the values $T_{n}(z)$ are $d$-closed for any $n$ and $z \in Z$, then each $z \in Z_{1}$ satisfies (b).

\section{Applications to VARiational PRinciples}

In this section we show how the above general scheme applies to several well known variational principles. The framework is the following: we are given a completely regular topological space $X$, a subspace $X_{1} \subset X$ whose topology inherited from $X$ is metrizable by a complete metric $d$, and a Banach space $\left(Z,\|\cdot\|_{Z}\right)$ of real-valued continuous functions on $X$ which are bounded on $X_{1}$ and satisfy:

(H1) There is $M>0$ such that for all $g \in Z, M\|g\|_{Z} \geq \sup \left\{|g(x)|: x \in X_{1}\right\}$. Observe that the functions from $Z$ need not, in general, be bounded on $X$.

The space $Z$ will serve as a space of perturbation functions. More precisely, the question we are concerned with reads: given a function $f: X_{1} \rightarrow \mathbb{R} \cup\{+\infty\}$ which is proper, lower semicontinuous and bounded from below on $X_{1}$, what are the descriptive properties of the set $\{g \in Z$ : the perturbed minimization problem $\left(X_{1}, f+g\right)$ is well-posed $\}$.

To deal with this question, we shall follow the scheme developed in the previous section with the sequence of set-valued mappings $\left\{T_{n}: Z \rightrightarrows X_{1}\right\}_{n \geq 1}$ defined by

$$
T_{n}(g):=\left\{x \in X_{1}: f(x)+g(x) \leq \inf _{X_{1}}(f+g)+1 / n\right\}, \quad g \in Z, \quad n \geq 1,
$$

which for any $g \in Z$ gives, as value of the limit mapping, the minimum points of $f+g$ in $X_{1}$ :

$$
T(g)=\bigcap_{n=1}^{\infty} T_{n}(g)=\left\{x \in X_{1}: f(x)+g(x)=\inf _{X_{1}}(f+g)\right\}, \quad g \in Z .
$$

Given $A \subset X$, a proper function $h: X \rightarrow \mathbb{R} \cup\{+\infty\}$ which is bounded from below on $A$ and $\alpha>0$, we denote by $S(A, h, \alpha)$ the sub-level set

$$
S(A, h, \alpha):=\left\{x \in A: h(x) \leq \inf _{A} h+\alpha\right\} .
$$

So, $T_{n}(g)=S\left(X_{1}, f+g, 1 / n\right)$. Obviously, $\left\{T_{n}\right\}_{n \geq 1}$ is a decreasing sequence and all the sets $T_{n}(g)$ are nonempty and closed in $X_{1}$. To apply Theorem 2.2 we need to find conditions assuring that this sequence is fragmented by the metric $d$. The following proposition gives such conditions. 
Proposition 3.1. In the above framework, sufficient conditions (in decreasing order of generality) in order for the sequence $\left\{T_{n}: Z \rightrightarrows X_{1}\right\}_{n \geq 1}$ to be fragmented by $d$ are the following:

(F) For each $h: X_{1} \rightarrow \mathbb{R} \cup\{+\infty\}$ which is proper, lower semicontinuous and bounded from below in $X_{1}$ and every $\varepsilon>0$ there are $g \in Z$ and $\alpha>0$ so that $\|g\|_{Z} \leq \varepsilon$ and $d-\operatorname{diam} S\left(X_{1}, h+g, \alpha\right)<\varepsilon$.

(S) For every $\varepsilon>0$ there is $\beta>0$ such that for each $x_{1} \in X_{1}$ there exists $g \in Z$ with $\|g\|_{Z}=1$ and $g\left(x_{1}\right)+\beta \leq \inf \left\{g(x): x \in X_{1} \backslash B_{X_{1}}\left(x_{1}, \varepsilon\right)\right\}$.

Proof. First we show that (F) implies that $\left\{T_{n}\right\}_{n \geq 1}$ is fragmented by $d$. Let $U$ be a nonempty open subset of $Z$ and $\varepsilon>0$. Take $g_{0} \in U$ and $\gamma>0$ with $B_{Z}\left(g_{0}, \gamma\right) \subset U$. According to (F) there is $g \in Z$ such that $\|g\|_{Z} \leq \bar{\varepsilon}:=\min \{\varepsilon, \gamma / 2\}$ and $\alpha>0$ with $d-\operatorname{diam} S\left(X_{1}, f+g_{0}+g, \alpha\right)<\bar{\varepsilon}$. On the other hand, because of (H1) it is well known (and straightforward to see) that there is a positive $\delta<\gamma / 2$ so that $S\left(X_{1}, f+g^{\prime}, \alpha / 2\right) \subset S\left(X_{1}, f+g_{0}+g, \alpha\right)$ for any $g^{\prime} \in B_{Z}\left(g_{0}+g, \delta\right)$. Thus, $d-\operatorname{diam} T_{n^{\prime}}\left(U^{\prime}\right)<\varepsilon$ for $U^{\prime}=B\left(g_{0}+g, \delta\right) \subset U$ and $n^{\prime}$ such that $1 / n^{\prime}<\alpha / 2$.

Next, we show that $(\mathrm{S})$, which is a kind of separation of the points and closed sets of $X_{1}$ by functions from $Z$, implies (F). Let $h: X \rightarrow \mathbb{R} \cup\{+\infty\}$ be as in (F) and $\varepsilon>0$. Take $\beta>0$ as given by (S), put $\alpha:=\varepsilon \beta / 2$ and pick $x_{1} \in X_{1}$ so that

$$
h\left(x_{1}\right)<\inf _{X_{1}} h+\alpha .
$$

Now let $g \in Z$ be the function provided by (S) for the point $x_{1}$. Set $g_{\varepsilon}:=\varepsilon g$ and let $x \in S\left(X_{1}, h+g_{\varepsilon}, \alpha\right)$. We have

$$
\left(h+g_{\varepsilon}\right)(x) \leq\left(h+g_{\varepsilon}\right)\left(x_{1}\right)+\alpha<\inf _{X_{1}} h+g_{\varepsilon}\left(x_{1}\right)+2 \alpha ;
$$

hence $g(x)<g\left(x_{1}\right)+\beta$, so $x \in B_{X_{1}}\left(x_{1}, \varepsilon\right)$ according to (S). This shows that the set $S\left(X_{1}, f+g_{\varepsilon}, \alpha\right)$ is contained in $B_{X_{1}}\left(x_{1}, \varepsilon\right)$; hence its $d$-diameter is less than $2 \varepsilon$, while $g_{\varepsilon} \in Z$ with $\left\|g_{\varepsilon}\right\|_{Z}=\varepsilon$. The proof that (F) holds is complete.

As a result, we have the following variational principle:

Theorem 3.2. Let $X$ be a completely regular topological space, let $X_{1} \subset X$ be a completely metrizable subspace by a metric d and let $\left(Z,\|\cdot\|_{Z}\right)$ be a Banach space satisfying $(\mathrm{H} 1)$ and $(\mathrm{S})$ (or, more generally, (H1) and (F)). Suppose that $f: X_{1} \rightarrow \mathbb{R} \cup\{+\infty\}$ is proper, lower semicontinuous and bounded from below on $X_{1}$. Then, there is a dense $G_{\delta}$-subset $Z_{1}$ of $Z$ such that for any $g \in Z_{1}$ the perturbed minimization problem $\left(X_{1}, f+g\right)$ is well-posed.

Proof. As observed, $\left\{T_{n}\right\}_{n \geq 1}$ is a decreasing sequence and the sets $T_{n}(g)$ are nonempty and closed in $\left(X_{1}, \bar{d}\right)$. Moreover, it follows from Proposition 3.1 that this sequence is also fragmented by the metric $d$. Thus, by Theorem 2.2, there is a dense $G_{\delta}$-subset $Z_{1}$ of $Z$ so that for each $g \in Z_{1}$ we have the property (b) for the limit mapping (because the metric $d$ is complete). The property (b) for $g$ evidently implies the well-posedness of $\left(X_{1}, f+g\right)$.

The Deville-Godefroy-Zizler variational principle is a consequence of Theorem 3.2 ;

Theorem $3.3(\overline{D G Z 1}, \overline{D G Z 2}])$. Let $(X,\|\cdot\|)$ be a Banach space and let $\left(Z,\|\cdot\|_{Z}\right)$ be a Banach space of continuous bounded functions in $X$ satisfying $(\mathrm{H} 1)$ with $X_{1}=X$ 
and the following additional properties:

(H2) For any $g \in Z$ and $x \in X$ the function $g_{x}: y \in X \mapsto g(x+y)$ is in $Z$ and, moreover, $\left\|g_{x}\right\|_{Z}=\|g\|_{Z}$.

(H3) For any $g \in Z$ and $t \in \mathbb{R}$ the function $x \in X \mapsto g(t x)$ is in $Z$.

(H4) There exists a bump function in $Z$, i.e. a function $b: X \rightarrow \mathbb{R}$ with a bounded nonempty support.

Suppose that $f: X \rightarrow \mathbb{R} \cup\{+\infty\}$ is proper, lower semicontinuous and bounded from below on $X$. Then, there is a dense $G_{\delta}$-subset $Z_{1}$ of $Z$ such that for any $g \in Z_{1}$ the perturbed minimization problem $(X, f+g)$ is well-posed.

Proof. The result will follow from Theorem 3.2 with $X_{1}=X$ once we have checked that the properties (H2)-(H4) imply the separation property (S). First note that, because of (H2) and (H3), we may suppose that the support of the bump $b$ given by (H4) lies in the (open) unit ball $B_{X}(0,1)$ of $X$ and that $b(0)=1$. Let $\varepsilon>0$ be arbitrary. Consider the function $b_{\varepsilon}(\cdot)=b(\cdot / \varepsilon)$ which is in $Z$ and whose support lies in the open ball $B_{X}(0, \varepsilon)$. Put $\beta:=1 /\left\|b_{\varepsilon}\right\|_{Z}$. Now let $x_{1} \in X$ be arbitrary. The function $g(\cdot):=-\beta b_{\varepsilon}\left(\cdot-x_{1}\right)$ is in $Z,\|g\|_{Z}=\beta\left\|b_{\varepsilon}\right\|_{Z}=1, g\left(x_{1}\right)+\beta=0$ and $g$ is zero outside the ball $B_{X}\left(x_{1}, \varepsilon\right)$. Hence, (S) is verified.

Let us mention that particular important cases when one has the conditions (H1)-(H4) fulfilled include the family of all bounded continuous functions in $X$, the family of all bounded Lipschitz functions in $X$ and the family of all bounded Lipschitzian $C^{1}$-smooth functions in a Banach space admitting a Lipschitzian $C^{1}$ smooth bump with the corresponding norms. This allows many applications of the Deville-Godefroy-Zizler variational principle, among them the possibility to derive (the weak form) of the Ekeland variational principle and to prove existence of solutions to Hamilton-Jacobi equations (see [DGZ1, DGZ2 for more details).

Further, we show how (one part of) the generic variational principle of Coban,

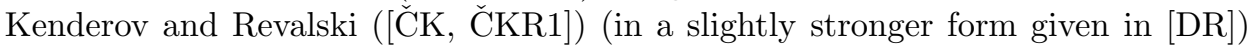
follows also by Theorem 3.2 .

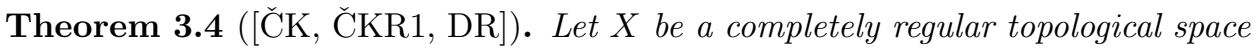
which contains a dense completely metrizable subspace $X_{1}$. Let $f: X \rightarrow \mathbb{R} \cup\{+\infty\}$ be a proper, lower semicontinuous and bounded from below function in $X$ which is continuous at any point of $\operatorname{dom} f$. Then the set $\left\{g \in C_{b}(X):(X, f+g)\right.$ is well-posed $\}$ contains a dense $G_{\delta}$-subset of the space $\left(C_{b}(X),\|\cdot\|_{\infty}\right)$.

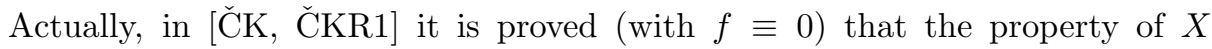
to contain a dense completely metrizable subspace characterizes the fact that the well-posed problems in $C_{b}(X)$ form a residual subset of $C_{b}(X)$. A strengthening of the above theorem (as well as of the Deville-Godefroy-Zizler principle) to get a stronger (than the first Baire category) property of smallness of the complement of the well-posed problems, the so-called $\sigma$-porosity, was done in $\mathrm{DR}$.

Proof. First, observe that because of the assumptions on $f$ and $X_{1}$ the domain $\operatorname{dom} f$ of $f$ is open in $X$ and moreover $\inf _{X}(f+g)=\inf _{X_{1}}(f+g)$ for any $g \in C_{b}(X)$. In particular, $f$ is proper on $X_{1}$ too. The space $Z=C_{b}(X)$ supplied with the supnorm $\|\cdot\|_{\infty}$ satisfies (H1), and because $X$ is completely regular, the separation property (S) is satisfied too. Thus, by Theorem 3.2 there is a dense $G_{\delta}$-subset $Z_{1}$ of $C_{b}(X)$ so that for each $g \in Z_{1}$ the problem $\left(X_{1}, f+g\right)$ is well-posed. We show 
that for $g \in Z_{1}$ the minimization problem $(X, f+g)$ is well-posed too. Indeed, take $g \in Z_{1}$ and let $x_{0} \in X_{1}$ be the unique minimum of the problem $\left(X_{1}, f+g\right)$. First, since every minimizing net for $\left(X_{1}, f+g\right)$ also converges to $x_{0}$ and $X_{1}$ is dense in $X$, the minimization problem $(X, f+g)$ cannot have other solutions than $x_{0}$. Therefore, $\left\{x_{0}\right\}=\bigcap_{k \geq 1} O_{k}$, where $O_{k}:=\left\{x \in X: f(x)+g(x)<\inf _{X}(f+g)+1 / k\right\}$. We show, in addition, that the family of open sets $\left\{O_{k}\right\}_{k \geq 1}$ is a (countable) local base for $x_{0}$ in $X$. From this the well-posedness of $(X, f+g)$ follows immediately.

Take an open set $V$ of $X$ so that $V \ni x_{0}$. Since $X$ is completely regular there is an open set $W$ in $X$ with $W \ni x_{0}$ and the closure $\bar{W}$ is contained in $V$. We claim that there is $k_{0}$ so that $O_{k_{0}} \subset \bar{W} \subset V$. Otherwise, for any $k \geq 1$ we have $O_{k} \backslash \bar{W} \neq \emptyset$ and since the latter set is open in $X$ and $X_{1}$ is dense in $X$ we obtain a sequence $x_{k} \in\left(O_{k} \backslash \bar{W}\right) \cap X_{1}, k \geq 1$. Evidently $\left\{x_{k}\right\}_{k \geq 1} \subset X_{1}$ is a minimizing sequence for $\left(X_{1}, f+g\right)$ which does not converge to $x_{0}$. This contradiction completes the proof.

The above generic variational principle has been used for the study of the differ-

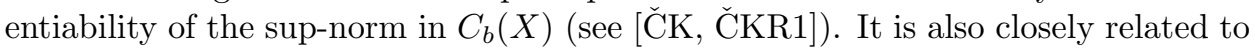
the existence of (special) winning strategies for one of the players in the Banach-

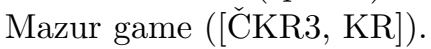

Next, let us see that the well known Stegall variational principle also fits the above general scheme. Here again $(X,\|\cdot\|)$ denotes a real Banach space. Recall that when $x^{*}$ is an element of the dual $X^{*}$ of $X$ the sets $S\left(A, x^{*}, \alpha\right)$ are called slices in $A \subset X$. A subset $C$ of a Banach space $X$ is said to satisfy the Radon-Nikodým property (in short RNP) if every bounded subset $A$ of $C$ is dentable. The set $A$ is dentable if $A$ admits slices of arbitrarily small (norm-) diameter.

Theorem 3.5 ( $[\mathrm{St}]$ ). Let $C$ be a nonempty bounded closed convex subset of a Banach space $X$. Suppose that $C$ satisfies $R N P$ and let $f: C \rightarrow \mathbb{R} \cup\{+\infty\}$ be proper, lower semicontinuous and bounded from below on $C$. Then the set $\left\{x^{*} \in\right.$ $X^{*}:\left(C, f+x^{*}\right)$ is well-posed $\}$ contains a dense $G_{\delta}$-subset of $X^{*}$.

Let us recall that, when the maximization problem $\left(C, x^{*}\right)$ for some $x^{*} \in X^{*}$ is well-posed, then the unique maximum in $C$ is said to be strongly exposed (by $x^{*}$ ) and the functional $x^{*}$ is called a strongly exposing functional for $C$. An immediate consequence of the Stegall variational principle (with $f \equiv 0$ ) is that the set of strongly exposing functionals for a nonempty closed convex bounded subset $C \subset X$ with RNP is a residual subset of $X^{*}$. Another important consequence is that every nonempty closed bounded convex set with RNP in $X$ is the closed convex hull of its strongly exposed points (see e.g. Phelps [Ph], Theorem 5.20).

One way to prove the above result passes by the following key fact (see e.g. Phelps [ $\underline{\mathrm{Ph}}]$, Proof of Theorem 5.15, page 90).

Proposition 3.6. Let $C$ be a nonempty bounded closed convex subset of a Banach space $X$. If $C$ satisfies $R N P$, then for every $h: C \rightarrow \mathbb{R} \cup\{+\infty\}$ which is proper, lower semicontinuous and bounded from below on $C$ and every $\varepsilon>0$, there are $x^{*} \in X^{*}$ with $\left\|x^{*}\right\|<\varepsilon$ and $\alpha>0$ such that $\operatorname{diam} S\left(C, h+x^{*}, \alpha\right)<\varepsilon$.

Proof of Theorem 3.5. We may apply Theorem 3.2 with $X_{1}=C$ and $Z=X^{*}$ with its dual norm since (H1) is verified with $M=\sup \{\|x\|: x \in C\}$ and (F) holds according to Proposition 3.6 . 
Finally, as a further illustration of our approach, we show how the variational principle of Ioffe and Zaslavski follows readily from our general Theorem 2.2

Theorem 3.7 ([IZa $)$. Let $(X, d)$ be a complete metric space and let $Z$ be a Baire metric space such that for any $z \in Z$ there is associated a proper lower semicontinuous function $f_{z}: X \rightarrow[-\infty,+\infty]$ that satisfies the following hypothesis:

$(\mathcal{H})$ there is a dense subset $Z_{0}$ of $Z$ so that for any $z \in Z_{0}$, any $\varepsilon>0$ and any $\gamma>0$, there exist a nonempty open set $U \subset Z, x \in X, \alpha>0$ and $\eta>0$ such that $U$ belongs to the ball of radius $\varepsilon$ around $z$ and for any $u \in U$ one has:

(i) $\inf f_{u}>-\infty$;

(ii) if $y \in X$ is such that $f_{u}(y) \leq \inf f_{u}+\eta$, then $d(y, x) \leq \gamma$ and $\left|f_{u}(y)-\alpha\right| \leq \gamma$. Then, there exists a dense $G_{\delta}$-subset $Z_{1}$ of $Z$ so that for any $z \in Z_{1}$, the infimum of $f_{z}$ is finite and attained at a unique point $x \in X$. Moreover, the following kind of strong well-posedness is true for $f_{z}$ : if $z_{n} \rightarrow z$, then inf $f_{z_{n}}>-\infty$ eventually, and if $x_{n} \in X$ satisfy $f_{z_{n}}\left(x_{n}\right)-\inf f_{z_{n}} \rightarrow 0$, then $x_{n} \rightarrow x$.

Proof. By the hypothesis $(\mathcal{H})$ there is an open and dense set $Z^{\prime}$ of $Z$, so that for any $z \in Z^{\prime}$ one has $\inf f_{z}>-\infty$. Evidently $Z^{\prime}$ is a Baire subspace of $Z$. Define $T, T_{n}$ in $Z^{\prime}$ as above: $T(z):=\left\{x \in X: f_{z}(x)=\inf f_{z}\right\}$ and $T_{n}(z):=\{x \in X:$ $\left.f_{z}(x) \leq \inf f_{z}+1 / n\right\}, n \geq 1$. Obviously $\left\{T_{n}\right\}_{n \geq 1}$ is decreasing and the sets $T_{n}(z)$ are nonempty and closed in $X$ for any $n$ and $z \in Z^{\prime}$. Moreover, it is seen from $(\mathcal{H})$ that the sequence $\left\{T_{n}\right\}_{n \geq 1}$ is fragmented by the metric in $X$. By Theorem 2.2. there is a dense $G_{\delta}$-subset $Z_{1}$ of $Z^{\prime}$ each point of which satisfies (b). Since $Z^{\prime}$ is open and dense in $Z$, then $Z_{1}$ is dense $G_{\delta}$ in $Z$ as well. This completes the proof since the condition (b) implies also the required strong well-posedness.

The principle above has an additional conclusion for the continuity of the infimalvalue function. This continuity is usually fulfilled in all the applications. Let us mention that the above principle is applicable also to situations where one considers perturbations with a distance between them weaker than the uniform one (for example the uniform convergence on bounded sets). Various such applications, concerning convex problems, quadratic problems, optimal control problems, etc., could be found in [Za, ILR.

\section{ACKNOWLEDGEMENTS}

The authors are grateful to an anonymous referee for his/her remarks which led to an improvement of the presentation of both the notions and the results.

\section{REFERENCES}

[BPr] J.M. Borwein and D. Preiss, A smooth variational principle with applications to subdifferentiability and differentiability of convex functions, Trans. Amer. Math. Soc. 303(1987), 517-527. MR0902782 (88k:49013)

[BCFR] J.M. Borwein, L. Cheng, M. Fabian and J.P. Revalski, A one perturbation variational principle and applications, Set-Valued Anal., 12(2004), 49-60. MR2069351

[ChrK] J.P.R. Christensen and P.S. Kenderov, Dense strong continuity of mappings and the Radon-Nikodým property, Math. Scand., 54(1984), 70-78. MF 0753064 (85k:46015)

[ČK] M.M. Čoban and P.S. Kenderov, Dense Gâteaux differentiability of the sup-norm in $C(T)$ and the topological properties of T, Compt. Rend. Acad. Bulg. Sci., 38(1985), 1603-1604. MF 0837262 (87h:46070)

[ČKR1] M.M. Coban, P.S. Kenderov and J.P. Revalski, Generic well-posedness of optimization problems in topological spaces, Mathematika, 36(1989), 301-324. MR1045790 (91c:90119) 
[ČKR2] M.M. Čoban, P.S. Kenderov and J.P. Revalski, Densely defined selections of multivalued mappings, Trans. Amer. Math. Soc., 344(1994), 533-552. MR1154539 (94k:54039)

[ČKR3] M.M. Čoban, P.S. Kenderov and J.P. Revalski, Topological spaces related to the BanachMazur game and to the generic well-posedness of optimization problem, Set-Valued Anal., 3(1995), 263-279. MR1353413 (96h:90172)

[DGZ1] R. Deville, G. Godefroy and V. Zizler, A smooth variational principle with applications to Hamilton-Jacobi equations in infinite dimensions, J. Funct. Anal., 111 (1993), 197-212. MF 1200641 (94b:49010)

[DGZ2] R. Deville, G. Godefroy and V. Zizler, Smoothness and renormings in Banach spaces, Pitman monographs and Surveys in Pure and Appl. Math., Longman Scientific \& Technical, 1993. MR1211634 (94d:46012)

[DR] R. Deville and J.P. Revalski, Porosity of ill-posed problems. Proc. Amer. Math. Soc., 128 (2000), 1117-1124. MR1636942 (2000i:49033)

[Ek1] I. Ekeland, On the variational principle, J. Math. Anal. Appl., 47(1974), 324-353. MF 0346619 (49:11344)

[Ek2] I. Ekeland, Nonconvex minimization problems, Bull. Amer. Math. Soc., 1(1979), 443474. MR 0526967 (80h:49007)

[HJT] R.W. Hansell, J.E. Jayne and M. Talagrand, First class selectors for weakly upper semicontinuous multivalued maps in Banach spaces, J. Reine Angew. Math., 361(1985), 201220. Mr $0807260(87 \mathrm{~m}: 54059 \mathrm{a})$

[ILR] A.D. Ioffe, R. Lucchetti and J.P. Revalski, A variational principle for problems with functional constraints, SIAM J. Optim., 12, No.2(2001), 461-478. MR1885571 (2002m:49042)

[IZa] A.D. Ioffe and A.J. Zaslavski, Variational principles and well-posedness in optimization and calculus of variations. SIAM J. Control Optim., 38 (2000), 566-581. MR 1741153 (2000m:49031)

[JNRo] J. E. Jayne, I. Namioka and C. A. Rogers, Topological properties of Banach spaces, Proc. London Math. Soc., (3) 66(1993), 651-672. MR,1207552 (94c:46041)

[JRo] J.E Jayne and C.A. Rogers, Borel selectors for upper semi-continuous set-valued maps, Acta Math., 155(1985), 41-79. MR0793237 (87a:28011)

[KMo] P. S. Kenderov and W. B. Moors, Fragmentability and sigma-fragmentability of Banach spaces, J. London Math. Soc., 60(1999), 203-223. MR.1721825 (2001f:46025)

[KR] P.S. Kenderov and J.P. Revalski, The Banach-Mazur game and generic existence of solutions to optimization problems, Proc. Amer. Math. Soc., 118(1993), 911-917. MF 1137224 (93i:49012)

[LW] P.D. Loewen, X.F. Wang, A generalized variational principle, Can. J. Math., 53(6)(2001), 1174-1193. MR1863847 (2002g:49029)

[Ph] R.R. Phelps, Convex Functions, Monotone Operators and Differentiability, Lect. Notes in Math. \# 1364, Springer Verlag, Berlin, 1993. MR.1238715 (94f:46055)

[Ri1] N.K. Ribarska, Internal characterization of fragmentable spaces, Mathematika, 34(1987), 243-257. MR 0933503 (89e:54063)

[Ri2] N.K. Ribarska, The dual of a Gâteaux smooth Banach space is weak star fragmentable, Proc. Amer. Math. Soc., 114(1992), 1003-1008. MR1101992 (92g:46020)

[St] C. Stegall, Optimization of functions on certain subsets of Banach spaces, Math. Ann., 236(1978), 171-176. MR0503448 (80a:46022)

Laboratoire AOC, Département de Mathématiques, Université des Antilles et de la Guyane, 97159 Pointe-À-Pitre, France

E-mail address: marc.lassonde@univ-ag.fr

Institute of Mathematics and Informatics, Bulgarian Academy of Sciences, Acad. G. Bonchev Street, Block 8, 1113 Sofia, Bulgaria

E-mail address: revalski@math.bas.bg 